\title{
Natural Characteristics Analysis of Frame of 1780 Tandem Rolling Mill
}

\section{Dechen Zhang and Yanping Sun}

College of Mechanical Engineering and Automation, University of Science and Technology Liaoning, Anshan, Liaoning, China, 114051

\section{Zhangsun6411@163.com}

Key words: frame, natural frequency, finite element method, structural dynamics method

\begin{abstract}
According to the vibration happened in rolling process of 1780 tandem rolling mill. Finite element method and structural dynamic method were used to analysis in this paper. The natural frequencies of the rack were calculated. The comparision between two methods were done. It was helpful for designing and reducing vibration of the rolling process.
\end{abstract}

\section{Introduction}

The frame is the important component of the rolling mill. Bearing pedestal and adjusting device of the roller were installed on the rack. Not only enough strength and stiffness, should the rack have, but to avoid the impact force caused by the resonant frequency. In order to make a solid theory foundation for designing and reducing vibration of the rack, modal analysis has been done in this paper. Natural Characteristics Analysis of the rack has also been done to understand the dynamic characteristic of the rack [1].In this paper the abnormal vibration of the fifth frame of the 1780 hot rolling mill were analyzed. The modal of the rack were studied by finite element method and analytic method $[2,3]$.

\section{Modal analysis of the rack by finite element method}

Since the analysis were not used to calculate the stiffness and strength but dynamic analysis, it is necessary to simplify some parts of the rack of the rolling mill. The complete model of the rolling mill was built to obtain more accurate results. Symmetry boundary conditions were used in some previous researches of modeling the rolling mill. Since the vibration modals were not all symmetrical, the entire model of rolling mill would more actually describe the vibration of rolling mill. In addition, the constraint conditions of the model was only limited to foot margin, and thus can simulate free oscillation in three dimensional spaces. The 3D-model was built based on Solidworks considering the complexity. Then, modal analysis was carried out based on ANSYS. The 8-node hexahedral element type solid45 was used to analyze the entire structure. The meshing grid of the rack was shown in figure 1.

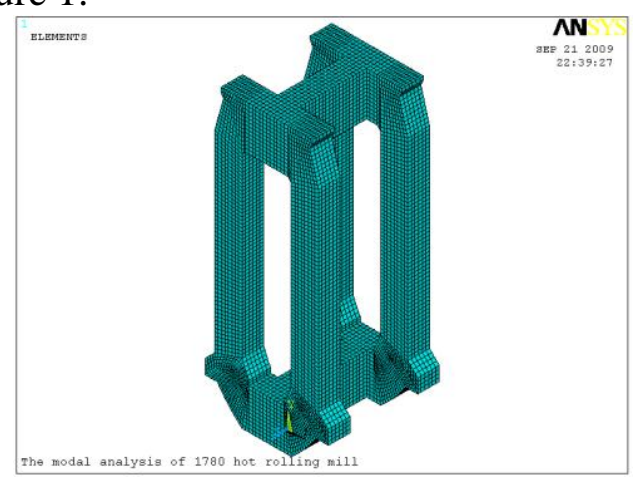

Fig. 1 The meshing grid of the rack

The main structural parameters of the rack were shown as follows:

Column height: $H=7.39 \mathrm{~m}^{2}$

Column cross-sectional area: $A_{1}=b \times h=0.94 \times 0.79 \mathrm{~m}^{2}$ 
Column moment of inertia:

$$
\begin{aligned}
& I_{1}=\frac{b h^{3}}{12}=0.94 \times 0.79^{3} / 12=0.0386 \mathrm{~m}^{4} \\
& I_{2}=\frac{b h^{3}}{12}=\frac{1}{12} \times 0.79 \times 0.94^{3}=0.0547 \mathrm{~m}^{4}
\end{aligned}
$$

\section{Conclusion of modal analysis of the rack}

The model was built by FEA method. According to the install of the rack and the force though the rolling process, the connection of the rack bolts were all rigid constraints. The $\mathrm{X}, \mathrm{Y}, \mathrm{Z}$ three directions of zero displacement constraints were imposed on the left and right of the foot bottom respectively. The natural frequencies of each rack were calculated and shown in table 1.

Table 1 The natural frequency of a single rack

\begin{tabular}{ccccccc}
\hline $\begin{array}{c}\text { order } \\
\text { natural }\end{array}$ & 1 & 2 & 3 & 4 & 5 & 6 \\
frequency $(\mathrm{Hz})$ & 10.330 & 14.452 & 19.349 & 43.620 & 52.208 & 56.168 \\
\hline $\begin{array}{c}\text { order } \\
\text { natural } \\
\text { frequency }(\mathrm{Hz})\end{array}$ & 7 & 8 & 9 & 10 & 11 & 13 \\
\hline
\end{tabular}

\section{Structural dynamic method}

Equivalent mass of the rack $M_{11}$ :

$M_{11}=2 M_{1}+M_{2}+4 \xi M_{3}$

The quality of Beam on the single rack: $M_{1}=3.56 \times 10^{4} \mathrm{~kg}$

The quality of Beam on the whole: $M_{2}=3.59 \times 10^{4} \mathrm{~kg}$

The quality of a single column rack: $M_{3}=4.2052 \times 10^{4} \mathrm{~kg}$

Focus on the quality factor: $\xi=0.371$

Flexibility of each column: $f=\frac{H^{3}}{12 E I}$

The results were calculated in Formula (1): $M_{11}=1.70 \times 10^{5} \mathrm{~kg}$

Flexibility of the rack: $f_{11}=0.25 f=1.488 \times 10^{-9} \mathrm{~m} / \mathrm{N}$

\section{Comparison between analytic method and FEM}

In this paper, many natural frequencies have been calculated. First, fifth and thirteenth order were chosen as numerical examples for analysis.

First-order natural frequency. The first order mode of two-piece rack was shown in Fig.2 (a). The equivalent mass method model of two-piece rack was shown in Fig.2 (b). The natural frequency of this order was most likely to vibrate in the structure of modal frequencies. It was relatively simple to calculate by the equivalent mass method.

The equivalent mass method was used to calculate the frequency. First, the original system was replaced by a single degree freedom system and the frequency was calculated by the formula. The lumped mass $\mathrm{m}$ and its directions were located to get the vibration frequency close to the minimum frequency of the original system. The value $\mathrm{m}$ are different according to the location of the equivalent mass. Once the location was known, the response $\mathrm{m}$ was determined. The calculation process was shown below. 


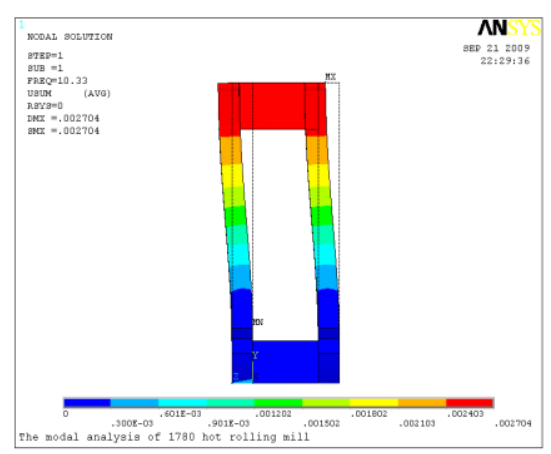

(a)

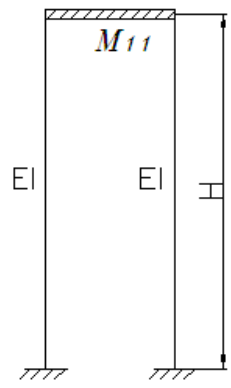

Fig. 2 The first order mode of the rack

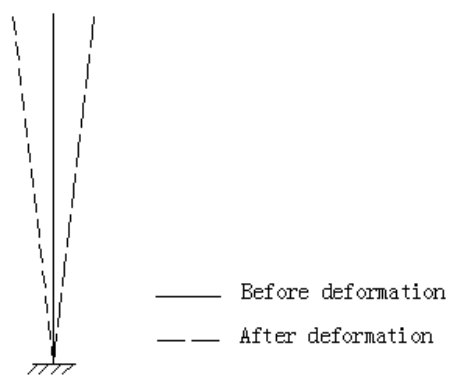

(b)

First-order natural frequency:

$$
\begin{aligned}
& \omega_{1}=\sqrt{\frac{1}{M_{11} \cdot f_{11}}}=62.87 \mathrm{rad} / \mathrm{s} \\
& f_{1}=\frac{\omega_{1}}{2 \pi}=10.01 \mathrm{~Hz}
\end{aligned}
$$

The results comparing analytical method with FEA method

The matching rate:

$$
\alpha=\frac{10.01}{10.33} \times 100 \%=96.9 \%
$$

5th-order natural frequency.5th-order modal shape and computation model in Fig.3 (a) and Fig.3 (b). For the rack of the 5th order mode, the modes of operating side of the column and the driving side of the column were symmetric. According to the actual deformation, the rack was computed by simplified into a beam one end fixed, the other end of hinged.

The main structural parameters and the calculation was shown as follows,

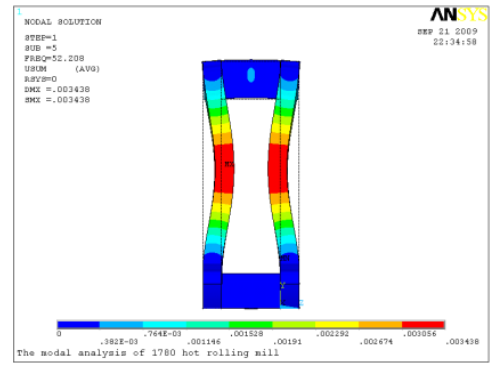

(a)

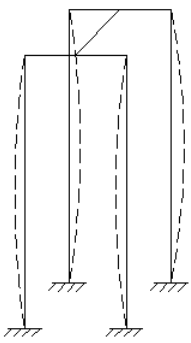

Fig. 3 The 5th order mode

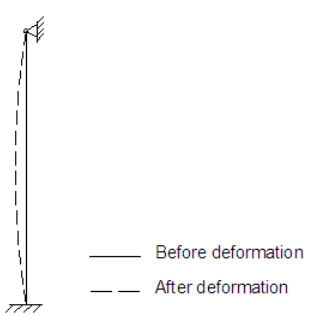

(b)

5th-order natural frequency:

$$
\begin{aligned}
& \omega_{5}=\frac{15.45}{H^{2}} \cdot \sqrt{\frac{E I_{1}}{\rho A_{1}}}=326.6 \mathrm{rad} / \mathrm{s} \\
& f_{5}=\frac{\omega_{5}}{2 \pi}=\frac{326.6}{6.28}=52.007 \mathrm{~Hz}
\end{aligned}
$$

The results comparing analytical method with FEA method,

The matching rate

$$
\alpha=\frac{52.007}{52.208} \times 100 \%=99.61 \%
$$

13th-order natural frequency (vertical vibration mode). The 13th-order mode of rack was shown in Fig.4 (a). The equivalent mass method model of rack was shown in Fig.4 (b). The vertical vibration mode in 13th-order mode of the rack occurred $[4,5]$. According to the modal of order 13, the mode was simplified of a single degree of freedom - spring system. In order to get the natural frequency, the calculation was shown as follows, 


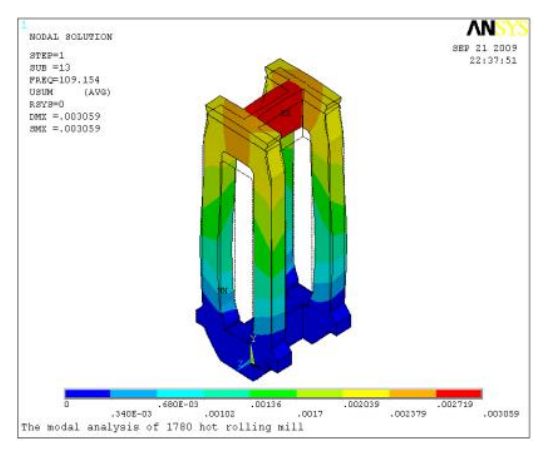

(a)

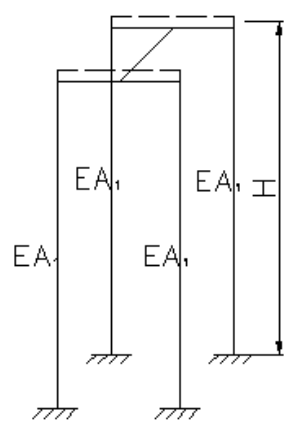

Fig. 4 The 13 th order mode

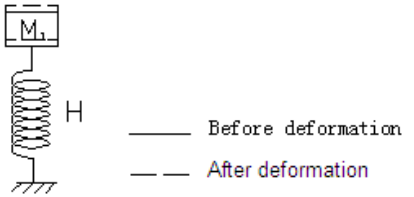

(b)

Equivalent mass $M_{11}$ of the rack

Focus on the quality factor $\xi=0.333$.

$M_{11}=1.631 \times 10^{5} \mathrm{~kg}$

The equivalent stiffness $K$

$A=4 A_{1}=4 \times 0.94 \times 0.79=2.9704 \mathrm{~m}^{2}$

$K=\frac{E A_{1}}{H}=8.039 \times 10^{10} \mathrm{~N} / \mathrm{m}$

where, $A$ are the Cross-sectional area of the rack and the column.

The 13th-order natural frequency $f_{13}$ as (vertical vibration)

$\omega_{13}=\sqrt{\frac{K}{M_{11}}}=702.1 \mathrm{rad} / \mathrm{s} \quad f_{13}=\frac{\omega_{13}}{2 \pi}=111.8 \mathrm{~Hz}$

The results comparing analytical method with FEA method,

The matching rate:

$$
\alpha=\frac{111.8}{108.663} \times 100 \%=102.88 \%
$$

\section{Conclusions}

The modal of the rack of 1780 hot rolling mill was analyzed by the FEA method and analytical method to get its natural frequency and vibration mode. It was helpful to understand the kinetics of the rack. The results of the two methods were compared and conformed these two methods were correct. It would be useful to provide an accurate dynamic characteristics and practical method to solve the problem of structure engineering.

\section{References}

[1] Zou-jia Xiang, Xu Le-jiang. Cold mill vibration control system [M],Metallurgical Industry Press , Beijing , 1998

[2] Zou Jia-xiang, Rolling machine [M],Metallurgical Industry Press , Beijing ,2000

[3] YUKIOK, YASUHIROS, NOBUON, et al. Analysis of chatter in tandem cold rolling mill [J]. ISIJ International , 2003,43:77-84

[4] Fu-xiang Hou, Jie Zhang, Jian-guo Cao and Xiao-lu Shi. Review of Chatter Studies in Cold Rolling,Journal of Iron and Steel Research,2007,19(10):6-10.

[5] Guanghong Zhu, Sen Bao, Jie Zhou, Liandong Fan and Rui-wen Wang. Research on the vibration of hot rolling mill.Reseah on Iron and Steel.2010,38:42-45. 